\title{
The Characteristic of Auditory Function and Cochlear Pathophysiology in a Noise-exposed Cohort: A Cross-sectional \\ Study
}

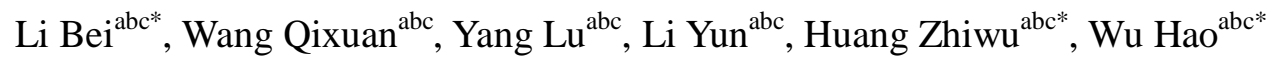

${ }^{\mathrm{a} D e p a r t m e n t}$ of Otolaryngology-Head and Neck Surgery, Shanghai Ninth People's

Hospital, Shanghai Jiao Tong University School of Medicine, China

${ }^{b}$ Ear Institute, Shanghai Jiao Tong University School of Medicine, Shanghai, China,

${ }^{\mathrm{c} S h a n g h a i}$ Key Laboratory of Translational Medicine on Ear and Nose Diseases,

China

Bei Li and Qixuan Wang contributed equally to this paper 


\begin{abstract}
Objectives: To determine the characteristic of auditory perception and cochlear pathophysiology in individuals with long-term occupational noise-exposure.

Design: Young workers with occupational noise-exposure from a shipyard were recruited for participation in the current study. Age-matched workers in the same shipyard who had no noise-exposure were enrolled in the control group. The speech-in-noise scores, gap detection thresholds and SP/AP values were tested and compared between the two groups. The correlations of both the speech-in-noise scores and the gap detection threshold with the SP/AP value were calculated and analyzed.

Results: Our results demonstrated that even within the normal auditory threshold, individuals with occupational noise exposure showed lower speech-in-noise scores and higher gap detection threshold and SP/AP values. Speech-in-noise score was correlated SP/AP value. The electrocochleography values showed no significant correlation with the gap detection threshold.
\end{abstract}

Conclusion: The result suggested that noise-induced cochlear synaptopathy probably occurs in humans with occupational noise exposure. However, they also implied that the mechanism of auditory dysfunction in humans is more complicated than that in experimental animals.

Key words: Cochlear synaptopathy; Hidden hearing loss; Occupational noise exposure; Speech-in-noise; Gap detection threshold 


\section{INTRODUCTION}

In the ENT clinic, patients often complain of hearing problems with pure-tone hearing thresholds within normal ranges. This is called clinical hidden hearing loss (cHHL). Difficulty hearing in noisy environments and the fast speech condition are the most common hearing defects in these individuals. It is challenging to unearth the evidence and the function of hearing impairment in cHHL individuals with only the regular clinical hearing test.

Recently, animal studies showed that noise-induced damage to the synapse between inner hair cells and type I afferent auditory nerve fibers may occur in the absence of a permanent threshold shift (Furman et al. 2013; Kujawa et al. 2009; Valero et al. 2017). These characteristic morphologic changes in the cochlea are termed cochlear synaptopathy, and this sort of hearing impairment is termed noise-induced hidden hearing loss (nHHL). Along with synapses and afferent auditory nerve fiber injure, other coding deficiencies, including loudness and temporal resolution, were also observed. For instance, Shi et al. (2016) found that the click-evoked compound action potential (CAP) amplitude was reduced after noise exposure in nHHL guinea pigs. Song et al. (2016) showed that the click-evoked CAP amplitude decreased with prolonged peak latency. Other studies also reported a significant reduction in the auditory brainstem response (ABR) I wave amplitude in nHHL rodents (Furman et al. 2013; Kujawa and Liberman 2009; 
Lin et al. 2011). Although the loudness and temporal deficits in cHHL individuals with normal hearing are almost identical to those in noise-exposed nHHL rodents, there is a lack of convincing direct pathologic evidence that noise-exposed human beings have cochlear synaptopathy.

Due to the absence of cochlear pathologic evidence, some electrophysiological values, such as the ABR Wave I amplitude (Schaette et al. 2011; Stamper et al. 2015), the ABR wave V latency (Mehraei et al. 2016), the frequency-following response (FFR) (Plack et al. 2016) and the ratio of summating potential relative to the action potential (SP/AP) (Liberman et al. 2016), have been reported as indicators of cochlear synaptopathy. Some of the electrophysiological indicators showed that cochlear synaptopathy and auditory nerve fiber degeneration also occurred in the cHHL population. Stamper and Johnson (2015) reported a decrease in ABR Wave I amplitude as a function of noise exposure during the previous 12 months in normal-hearing human ears. Schaette and McAlpine (2011) found a significant reduction in amplitude of the ABR Wave I in human subjects with tinnitus and a normal audiogram. Liberman et al. (2016) reported that the value of SP/AP increased in the high-risk group. Even so, it remains controversial that which indicator was the most appropriate. For instance, Plack et al. (2016) and Mehraei et al. (2016) argued that the ABR Wave I amplitude shows high variability both between and within individuals, and it was so difficult to measure in humans that it was not recommended. Liberman et al. (2016) reported a larger SP/AP value in the high-risk group; however, no significant difference was found in Wave I amplitude between 
groups. In summary, the electrophysiological indicators used in noise exposure research in humans should be chosen deliberately to avoid unreliable findings.

The purpose of this study was twofold: (1) to explore whether noise-exposed individuals showed cHHL and cochlear synaptopathy and (2) to examine the correlation between cHHL and cochlear synaptopathy in those individuals. Studies have showed that other factors, including aging (Fischer et al. 2019; Johannesen et al. 2019; Parthasarathy et al. 2018; Wu et al. 2018) and ototoxic drugs (Liu et al. 2015; Liu et al. 2013) also cause cochlear synaptopathy. In humans, the pathogenesis and mechanism of cHHL are complicated and may include, for example, noise exposure history mixed with aging or ototoxicity. To differentiate the effect of noise exposure on the human auditory system, noise-exposed young workers from a shipyard were recruited for participation in the current study. The individuals enrolled in the hidden hearing loss (HHL) risk group underwent rigorous screenings. Age-matched workers in the same shipyard who had no noise exposure were enrolled in the control group. Both the speech-in-noise scores and gap detection threshold were investigated in two groups and used as indicators of cHHL. The SP/AP value was applied as the indicator of nHHL. The speech-in-noise scores, gap detection threshold and SP/AP value were compared between the two groups. Finally, the correlation of both the speech-in-noise scores and the gap detection threshold with the SP/AP value was calculated and analyzed.

\section{MATERIALS AND PROCEDURES}

\section{Study design and participants}


This was a cross-sectional observational study performed from August to October 2019 and obtained appropriate Institutional Review Board (IRB) approval. Translational Medicine Ethics Review Committee of Shanghai Ninth People's Hospital Affiliated to Shanghai Jiao Tong University, School of Medicine granted an exemption of full review. All participants signed an informed consent form before participating.

\section{Participant recruitment}

The individuals in the HHL risk group and the control group were recruited from the factory of a shipyard. Individuals were instructed to fill in a questionnaire, and sex, age, handedness, auditory disease and ototoxicity history, working department, working hours per week and working age data were collected. An otoscope examine was performed to check the external auditory canal and tympanic membrane for both ears. Hearing thresholds of both ears were evaluated in all participants for free. Pure-tone thresholds of 500, 1000, 2000, 3000, 4000, 6000, and $8000 \mathrm{~Hz}$ were tested. Enrollment criteria in the HHL risk group including (1) age between 20 and 40 years old, (2) no ear disease or ototoxicity history, (3) hearing threshold of 500, 1000, 2000, 3000, 4000, 6000, $8000 \mathrm{~Hz}$ at less than or equal to 25 $\mathrm{dB} \mathrm{HL}$, (4) more than or equal to 3 working years in the noisy department. Enrollment criteria in the control group were the same as those in the HHL risk group except that they had no noise exposure history or shooting habits. 


\section{Noise exposure measurement}

Occupational noise-exposed individuals were instructed to wear the Aihua ASV5910 type personal exposure dosimeter (Hangzhou Aihua Instruments Co., Ltd, China) while at work, based on the standards of IEC 61672:2002 and IEC 61252:2002, to measure the equivalent sound pressure level (Leq) over a continuous 8 hours (8:00 AM. to 4:00 PM), the maximum acoustic pressure level ( $\left.\mathrm{LF}_{\max }\right)$, and the minimum acoustic pressure level $\left(\mathrm{LF}_{\min }\right)$ during weekdays. Dosimeters were calibrated by the Aihua AWA6221A type acoustic calibrator based on the standards of IEC 60942:2003, and the data were then imported into a computer. The occupational noise Leq of 8 hours is $89.4 \pm 7.8$ decibel A Weighted (dBA).

\section{Speech-in-noise test}

The original speech material was the Mandarin version of the Hearing in Noise Test (MHINT). The MHINT contains 14 lists that contains 20 sentences each. Each sentence contains 10 key words. The scores are expressed as percentages of the key words that were heard correctly. The MHINT sentences were recorded with a male speaker. The stimuli were presented at $65 \mathrm{~dB}$ (A) and were delivered bilaterally through Sennheiser HD580 headphones. In sentence recognition in noise, speech-shaped noise was presented at $65 \mathrm{~dB}$ (A) bilaterally as speech signals. The noise began $500 \mathrm{~ms}$ before the sentence and continued for $500 \mathrm{~ms}$ after the sentence had finished.

All participants had no experience with any speech tests before this study. 
Before the formal test, participants practiced as many times as they wished and were provided feedback to become familiar with stimuli. In the formal test, they were instructed to repeat the sentences as accurately as possible. Each sentence was played only once, and no feedback was provided in the formal tests.

\section{Gap detection threshold test}

The gap detection threshold test was measured in a three-interval forced-choice procedure. For the gap marker, white noise was low-pass filtered at cutoff frequencies of 1, 2, and $4 \mathrm{kHz}$ via 3000th-order finite impulse response filter with an approximately $-116 \mathrm{~dB} /$ octave filter slope. In brief, a three-interval forced-choice program was run on MATLAB software (version 7.0). Three buttons were presented on a monitor to the participant who was asked to indicate which one of the three stimuli were different (i.e., which of the three stimuli was inserted with a gap).

Details of the gap detection threshold test may be found in Li. et al 2017 (2017).

\section{Electrocochleography recording}

Electrocochleography recordings were collected using a commercial device (Intelligent Hearing Systems, US) with Smart EP software. The electrode impedance values were all less than $5 \mathrm{k} \Omega$, and the interelectrode impedance was within $1 \mathrm{k} \Omega$. A silver electrode with cotton and electrode gel was applied as the reference electrode in the ear canal. An electromagnetically shielded insert earphone (ER-3) was applied 
to deliver click stimulation to the test ear at $90 \mathrm{~dB}$ nHL in alternating polarity at a rate of 7.1/sec. The recorded potentials were amplified by a factor of 50,000 and filtered with $10 \mathrm{~Hz}$ (high-pass) and $3000 \mathrm{~Hz}$ (low-pass) filters. Averaged responses over 512 sweeps were acquired. The SP/AP of each ear was determined separately by two experienced audiologists. The mean SP/AP value from the two audiologists was calculated for electrocochleography value.

\section{RESULTS}

\section{Demographic characteristics and the audiometry of the participants}

In total, 142 individuals were assessed for eligibility, and 120 were included in the study. There were 60 participants ( 24 females) in control group and 60 participants (27 females) in the HHL risk group. The mean (SD) ages of the participants were 28 (4) years for the control group and 28 (4) years for the HHL risk group. Independent-samples t-test showed that the age difference of the two groups was not significant $(t=-0.498, \mathrm{p}=0.620)$. Fig. 1 shows the audiograms of the participants in the control group and the HHL risk group.

\section{FIGURE 1}

\section{Auditory function feature of the two groups}

\section{Speech-in-noise}

Speech-in-noise scores for individuals in each group are showed in Figure 2. In the noise condition $(\mathrm{SNR}=0$ ), both the control group and the HHL risk group 
achieved scores associated with good performance. Mean (SD) scores of the participants were 95.72 (2.24) for the control group and 92.78 (4.12) for the HHL risk group. Independent-samples t-test showed that the control group performed significantly better than the HHL risk group $(\mathrm{t}=4.864, \mathrm{p}<0.001)$ (Fig. 2).

\section{FIGURE 2}

\section{Gap detection threshold}

The gap detection thresholds in the groups varied in terms of gap marker cutoff frequency, as shown in Figure 3. Generally, the gap threshold of the control group was lower than that of the HHL risk group at the same gap marker. Data derived from subjects with the same gap marker frequencies were analyzed by independent-samples t-test. A significant difference was only observed for the $4 \mathrm{kHz}$ gap marker $(\mathrm{t}=-2.790, \mathrm{p}=0.006)$. No significant difference was evident at the $2 \mathrm{kHz}$ gap marker $(\mathrm{t}=-0.764, \mathrm{p}=0.447)$ or $1 \mathrm{kHz}$ gap marker $(\mathrm{t}=-1.356, \mathrm{p}=0.178)($ Fig. 3).

\section{FIGURE 3}

\section{Cochlear function feature of the two groups}

\section{Electrocochleography}

The waveforms of the electrocochleography of 120 ears in either groups were fitting in Figure 4. The fitting curves showed the trend of the waveforms changed. The curves demonstrated that the AP value in the HHL risk group decreased more sharply than the SP value compared to the control group. Because the baseline of the 
electrocochleography waveform varied in each individual and the latency of the AP and SP in each individual also varied in a small-time window, the SP and AP values of the fitting curve were not applied for statistics. The mean SP and AP value of the 2 ears in each individual were plotted in Figure 5 separately. Two-sample $\mathrm{T}$ tests showed that there was no significant difference in two groups $\left(t_{\mathrm{SP}}=0.140, \mathrm{p}=0.889\right.$; $\left.\mathrm{t}_{\mathrm{AP}}=1.019, \mathrm{p}=0.310\right)$

\section{FIGURE 4}

\section{FIGURE 5}

The SP/AP ratio of both ears was collected, and the electrocochleography values were analyzed in three different ways. First, the mean SP/AP value of both ears was calculated and compared between the two groups. The independent-samples $\mathrm{t}$-test showed that the differences were significant $(\mathrm{t}=-2.592, \mathrm{p}=0.011)$. Second, the worse (higher) SP/AP values of the two ears in each individual were compared between the two groups. The independent-samples t-test showed that the differences were also significant $(t=-2.589, \mathrm{p}=0.011)$. Finally, the SP/AP values of the ear on the same as that of the handedness were compared. No significant differences were founded in the independent-samples $t$-test $(t=-1.486, p=0.140)$. Each SP/AP value comparison is displayed in Figure 6.

\section{FIGURE 6}

\section{Correlation analysis}


We explored the relationship between the speech-in-noise score, gap detection and the electrocochleography value by calculating Pearson correlations. For the electrocochleography values, the mean, worse and handedness sides were all calculated. The results showed that the speech-in-noise score was significantly correlated with the mean SP/AP value and the worse SP/AP value. None of the three electrocochleography values showed a significant correlation with the gap threshold. The results of the Pearson correlation analysis are shown in Tables 1 and 2 .

\section{TABLE 1}

\section{TABLE 2}

\section{DISCUSSION}

Our results demonstrated that even within the normal auditory threshold, individuals with occupational noise exposure showed lower speech-in-noise scores and higher gap detection thresholds and SP/AP values, which are indicative of cHHL and cochlear synaptopathy. The speech-in-noise scores were correlated with the SP/AP values. None of the three electrocochleography values showed a significant correlation with gap thresholds.

Recently, a variety of noninvasive measures have been used to determine whether noise-induced cochlear synaptopathy occurs in humans (N. Bramhall et al. 2015; N. F. Bramhall et al. 2017; Fulbright et al. 2017; Grinn et al. 2017; Grose et al. 2017; Guest et al. 2017; Guest et al. 2018; Liberman et al. 2016; Stamper and Johnson 2015; Valderrama et al. 2018). However, the results are conflicting. 
Postmortem temporal bone studies have demonstrated that cochlear synaptopathy and neural degeneration exist widely among humans (Makary et al. 2011; Viana et al. 2015), even in young adults (Wu et al. 2018). Animal studies have proven that aging, noise exposure and ototoxicity drugs all cause cochlear synaptopathy (Kujawa and Liberman 2009; Liu et al. 2015; Sergeyenko et al. 2013; Zhang et al. 2020). As a result, cochlear synaptopathy probably exists widely among humans, and multiple different mechanisms, such as aging, noise exposure and ototoxicity drugs, could be involved. Therefore, it is harder to find the main cause of cochlear synaptopathy in different people.

Whether noise exposure causes cochlear synaptopathy in the auditory system depends on the sound pressure level and the exposure time of the noise (Fernandez et al. 2015). Whether noise exposure is the major cause of cochlear synaptopathy depends on the noise exposure dose differences between the chosen populations. In previous studies, a self-reported questionnaire was applied for noise exposure dose estimation and grouping. The self-reported questionnaire was easier to complete but depended mainly on the subjective impressions of the individuals. Lacking the exact exposure dose could lead to unreliable consequences when the self-reported questionnaire is applied to estimate the degree of cochlear synaptopathy between different individuals. In other words, the comparison of electrophysiological characteristics between the high-risk and low-risk groups based on a self-reported questionnaire does not always achieve satisfactory results. In contrast, using definite occupational noise measurements and exposure years is more reliable. It could also 
highlight the primary mechanisms and other reasons that lead to cochlear synaptopathy. This is the main reason that we achieved significant results.

The second reason is that the participants we recruited in the study were particularly young and age-matched. As mentioned above, different mechanisms, such as aging, noise exposure and ototoxicity drugs, could play important roles in cochlear synaptopathy. Researchers have tried to determine the relative contributions of age and noise exposure in predicting measures of cochlear synaptopathy. However, the result was inconsistent with the predicted effects of synaptopathy (Prendergast et al. 2019). This implied that it is probably difficult to distinguish the effects of aging and noise exposure on cochlear synaptopathy in humans. Hence, it is easier to find a significant difference when the age range is narrowed, and the noise exposure dose is expanded between groups. In our study, the mean age in both control and HHL risk groups was approximately 28 years. Reports have shown that the negative effect of aging on cochlear synaptopathy occurs at an early age (Wu et al. 2018). The limited age range for study inclusion also restrained the aging effect on cochlear synaptopathy. This is also an important reason that we achieved significant results.

Our results confirmed the negative effect of noise exposure on speech-in-noise score and temporal resolution, such as the gap detection threshold. Correlation analysis also showed that speech-in-noise scores were significantly correlated with the indicator of cochlear synaptopathy. However, the underlying mechanism is rather complicated. The ability of speech perception in noise requires not only intact peripheral auditory function but also intact central auditory factors such as attention, 
working memory and language. Cochlear synaptopathy could be one of the main deficits in peripheral auditory function. Prior studies have demonstrated that noise exposure also has a negative effect on the central auditory pathway. For example, Dewey et al. reported that fMRI responses throughout the auditory system were greater in individuals with higher lifetime noise exposure levels than in controls with low lifetime noise exposure levels (Dewey et al. 2020).

In Conclusion, compared to the control group, young individuals with a normal auditory threshold and long-term occupational noise exposure showed lower speech-in-noise scores and a higher gap detection threshold. Significant higher SP/AP value was also found in the noise-exposed individuals and was correlated with speech-in-noise scores.

\section{ACKNOWLEDGMENT}

This research was supported by the National Natural Science Foundation of China (grant 81700903 to BL) and Shanghai Key Laboratory of Translational Medicine on Ear and Nose Diseases (14DZ2260300). 


\section{REFERENCES}

Bramhall, N., Ong, B., Ko, J., et al. (2015). Speech Perception Ability in Noise is Correlated with Auditory Brainstem Response Wave I Amplitude. J Am Acad Audiol, 26, 509-517.

Bramhall, N. F., Konrad-Martin, D., McMillan, G. P., et al. (2017). Auditory Brainstem Response Altered in Humans With Noise Exposure Despite Normal Outer Hair Cell Function. Ear Hear, 38, e1-e12.

Dewey, R. S., Francis, S. T., Guest, H., et al. (2020). The association between subcortical and cortical fMRI and lifetime noise exposure in listeners with normal hearing thresholds. Neuroimage, 204, 116239.

Fernandez, K. A., Jeffers, P. W., Lall, K., et al. (2015). Aging after noise exposure: acceleration of cochlear synaptopathy in "recovered" ears. J Neurosci, 35, 7509-7520.

Fischer, N., Johnson Chacko, L., Glueckert, R., et al. (2019). Age-Dependent Changes in the Cochlea. Gerontology, 1-7.

Fulbright, A. N. C., Le Prell, C. G., Griffiths, S. K., et al. (2017). Effects of Recreational Noise on Threshold and Suprathreshold Measures of Auditory Function. Semin Hear, 38, 298-318.

Furman, A. C., Kujawa, S. G., Liberman, M. C. (2013). Noise-induced cochlear neuropathy is selective for fibers with low spontaneous rates. J Neurophysiol, $110,577-586$.

Grinn, S. K., Wiseman, K. B., Baker, J. A., et al. (2017). Hidden Hearing Loss? No 
Effect of Common Recreational Noise Exposure on Cochlear Nerve Response Amplitude in Humans. Front Neurosci, 11, 465.

Grose, J. H., Buss, E., Hall, J. W., 3rd. (2017). Loud Music Exposure and Cochlear Synaptopathy in Young Adults: Isolated Auditory Brainstem Response Effects but No Perceptual Consequences. Trends Hear, 21, 2331216517737417.

Guest, H., Munro, K. J., Plack, C. J. (2017). Tinnitus with a normal audiogram: Role of high-frequency sensitivity and reanalysis of brainstem-response measures to avoid audiometric over-matching. Hear Res, 356, 116-117.

Guest, H., Munro, K. J., Prendergast, G., et al. (2018). Impaired speech perception in noise with a normal audiogram: No evidence for cochlear synaptopathy and no relation to lifetime noise exposure. Hear Res, 364, 142-151.

Johannesen, P. T., Buzo, B. C., Lopez-Poveda, E. A. (2019). Evidence for age-related cochlear synaptopathy in humans unconnected to speech-in-noise intelligibility deficits. Hear Res, 374, 35-48.

Kujawa, S. G., Liberman, M. C. (2009). Adding insult to injury: cochlear nerve degeneration after "temporary" noise-induced hearing loss. J Neurosci, 29, 14077-14085.

Li, B., Guo, Y., Yang, G., et al. (2017). Effects of Various Extents of High-Frequency Hearing Loss on Speech Recognition and Gap Detection at Low Frequencies in Patients with Sensorineural Hearing Loss. Neural Plast, 2017, 8941537.

Liberman, M. C., Epstein, M. J., Cleveland, S. S., et al. (2016). Toward a Differential Diagnosis of Hidden Hearing Loss in Humans. PLoS One, 11, e0162726. 
Lin, H. W., Furman, A. C., Kujawa, S. G., et al. (2011). Primary neural degeneration in the Guinea pig cochlea after reversible noise-induced threshold shift. $J$ Assoc Res Otolaryngol, 12, 605-616.

Liu, K., Chen, D., Guo, W., et al. (2015). Spontaneous and Partial Repair of Ribbon Synapse in Cochlear Inner Hair Cells After Ototoxic Withdrawal. Mol Neurobiol, 52, 1680-1689.

Liu, K., Jiang, X., Shi, C., et al. (2013). Cochlear inner hair cell ribbon synapse is the primary target of ototoxic aminoglycoside stimuli. Mol Neurobiol, 48, 647-654.

Makary, C. A., Shin, J., Kujawa, S. G., et al. (2011). Age-related primary cochlear neuronal degeneration in human temporal bones. J Assoc Res Otolaryngol, 12, 711-717.

Mehraei, G., Hickox, A. E., Bharadwaj, H. M., et al. (2016). Auditory Brainstem Response Latency in Noise as a Marker of Cochlear Synaptopathy. J Neurosci, $36,3755-3764$.

Parthasarathy, A., Kujawa, S. G. (2018). Synaptopathy in the Aging Cochlea: Characterizing Early-Neural Deficits in Auditory Temporal Envelope Processing. J Neurosci, 38, 7108-7119.

Plack, C. J., Leger, A., Prendergast, G., et al. (2016). Toward a Diagnostic Test for Hidden Hearing Loss. Trends Hear, 20.

Prendergast, G., Couth, S., Millman, R. E., et al. (2019). Effects of Age and Noise Exposure on Proxy Measures of Cochlear Synaptopathy. Trends Hear, 23, 
2331216519877301.

Schaette, R., McAlpine, D. (2011). Tinnitus with a normal audiogram: physiological evidence for hidden hearing loss and computational model. J Neurosci, 31, $13452-13457$.

Sergeyenko, Y., Lall, K., Liberman, M. C., et al. (2013). Age-related cochlear synaptopathy: an early-onset contributor to auditory functional decline. $J$ Neurosci, 33, 13686-13694.

Shi, L., Chang, Y., Li, X., et al. (2016). Cochlear Synaptopathy and Noise-Induced Hidden Hearing Loss. Neural Plast, 2016, 6143164.

Song, Q., Shen, P., Li, X., et al. (2016). Coding deficits in hidden hearing loss induced by noise: the nature and impacts. Sci Rep, 6, 25200.

Stamper, G. C., Johnson, T. A. (2015). Auditory function in normal-hearing, noise-exposed human ears. Ear Hear, 36, 172-184.

Valderrama, J. T., Beach, E. F., Yeend, I., et al. (2018). Effects of lifetime noise exposure on the middle-age human auditory brainstem response, tinnitus and speech-in-noise intelligibility. Hear Res, 365, 36-48.

Valero, M. D., Burton, J. A., Hauser, S. N., et al. (2017). Noise-induced cochlear synaptopathy in rhesus monkeys (Macaca mulatta). Hear Res, 353, 213-223.

Viana, L. M., O'Malley, J. T., Burgess, B. J., et al. (2015). Cochlear neuropathy in human presbycusis: Confocal analysis of hidden hearing loss in post-mortem tissue. Hear Res, 327, 78-88.

Wu, P. Z., Liberman, L. D., Bennett, K., et al. (2018). Primary Neural Degeneration in 
medRxiv preprint doi: https://doi.org/10.1101/2020.09.27.20202481; this version posted March 25, 2021. The copyright holder for this preprint (which was not certified by peer review) is the author/funder, who has granted medRxiv a license to display the preprint in perpetuity.

All rights reserved. No reuse allowed without permission.

the Human Cochlea: Evidence for Hidden Hearing Loss in the Aging Ear.

Neuroscience.

Zhang, W., Peng, Z., Yu, S., et al. (2020). Loss of Cochlear Ribbon Synapse Is a Critical Contributor to Chronic Salicylate Sodium Treatment-Induced Tinnitus without Change Hearing Threshold. Neural Plast, 2020, 3949161. 
Figure Legends

FIGURE 1. Group mean and standard deviation auditory thresholds (dB HL)

for the control group and the hidden hearing loss risk group

FIGURE 2. Group mean and standard deviation speech recognition scores for the control group and for the hidden hearing loss risk group

The $\mathrm{p}$ values less than 0.001 are indicated $* * *$

FIGURE 3. The individual and mean gap thresholds in the control group and the hidden hearing loss risk group.

The gap thresholds at different gap markers are showed in different panels.

The $\mathrm{p}$ values less than 0.01 are indicated $* *$.

FIGURE 4. Fitting curves of the electrocochleography of the control group and the hidden hearing loss risk group

The standard deviation of the control group was showed in gray and those of the hidden hearing loss risk group was showed in pink. Only the standard deviation below were showed.

FIGURE 5. Comparisons of the SP and AP values in the control group and the hidden hearing loss risk group

FIGURE 6. Comparisons of the SP/AP values in the control group and the hidden hearing loss risk group measured in 3 different ways.

The $\mathrm{p}$ values less than 0.05 are indicated $*$ 
A Hearing Threshold of the Left Ear

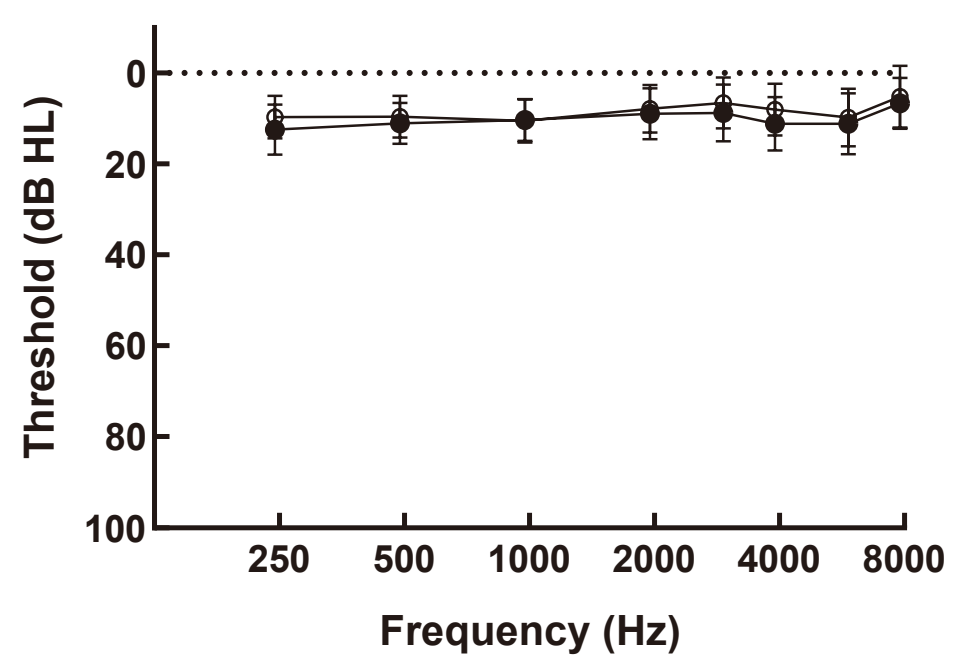

B Hearing Threshold of the Right Ear

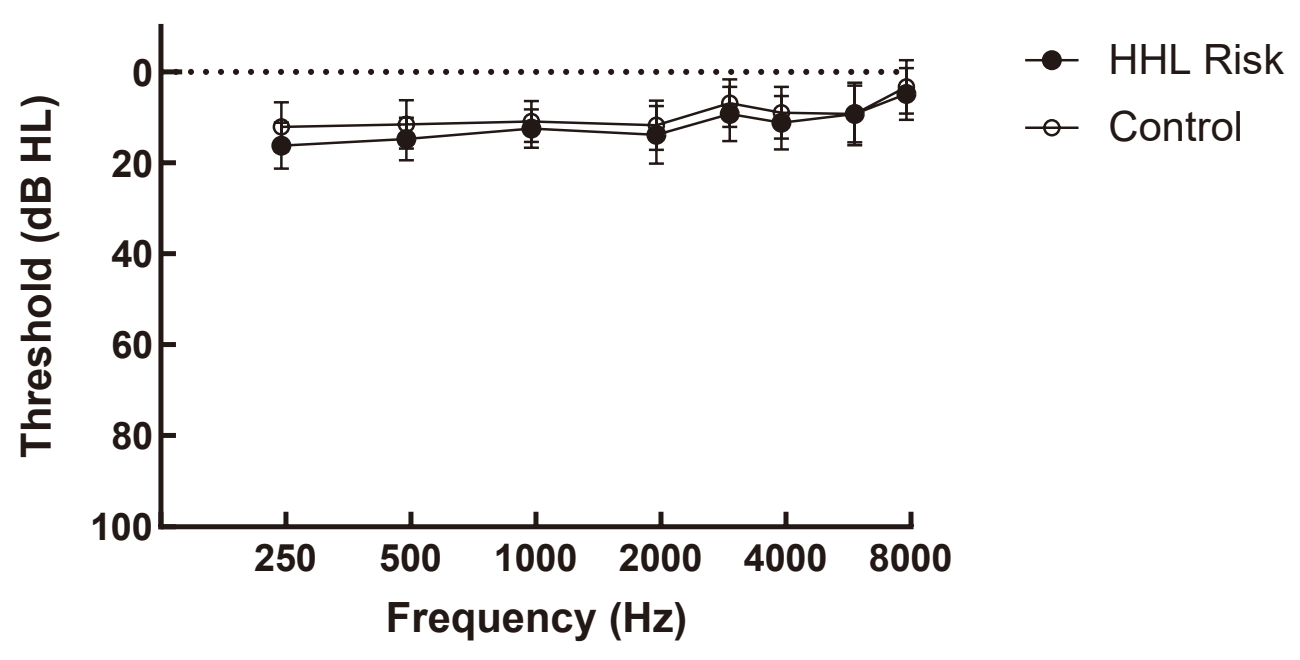




\section{Intact Speech}

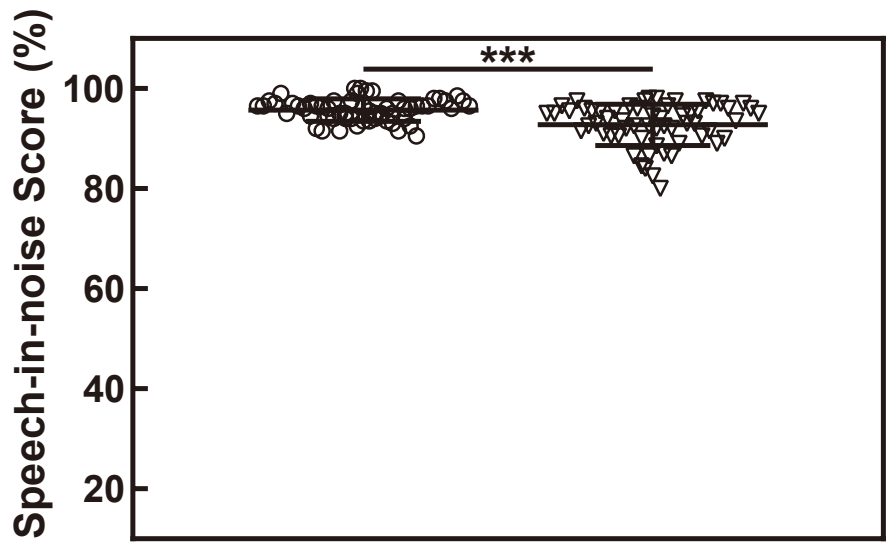

- Control

$\nabla$ HHL Risk 

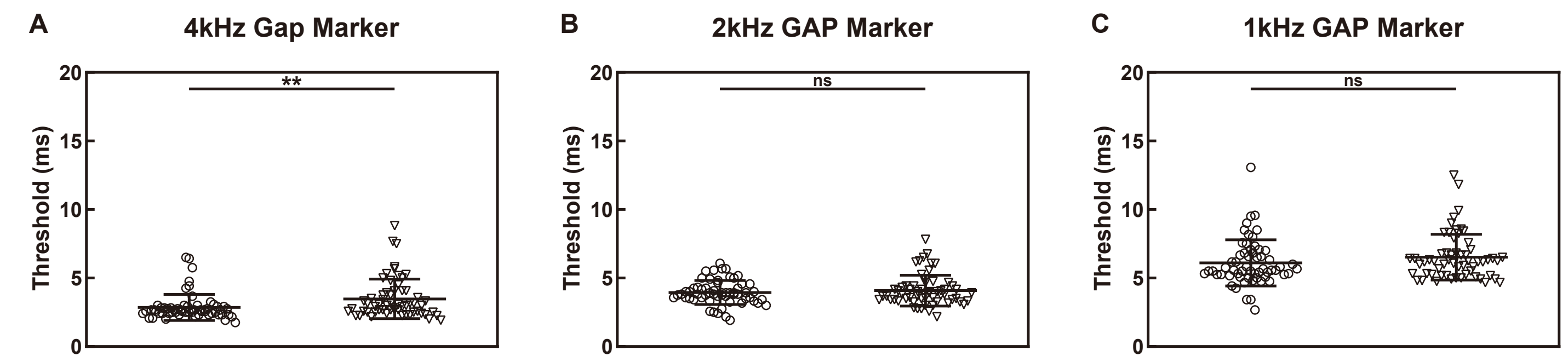


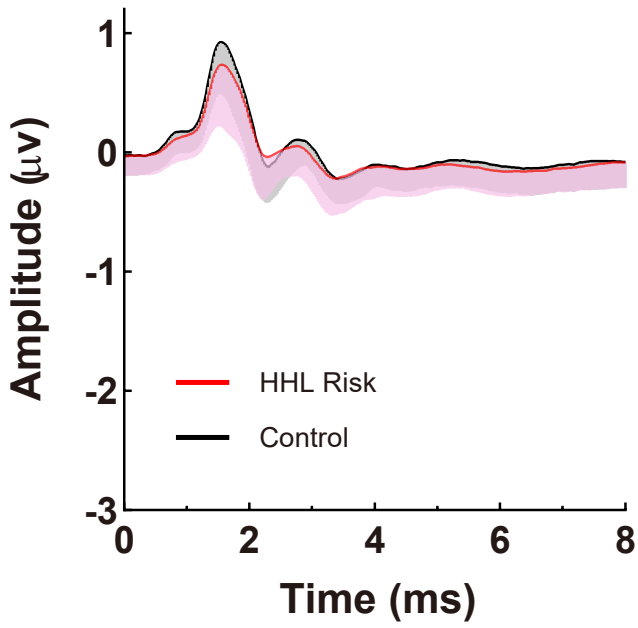



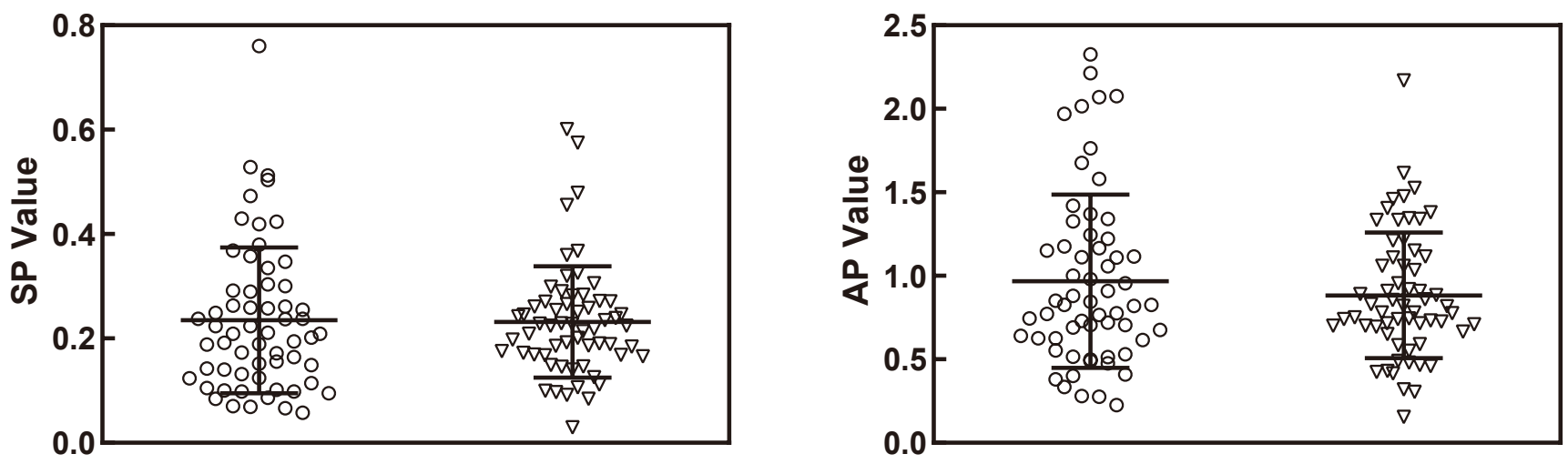

- Control

$\nabla$ HHL Risk 


\section{Mean SPIAP}

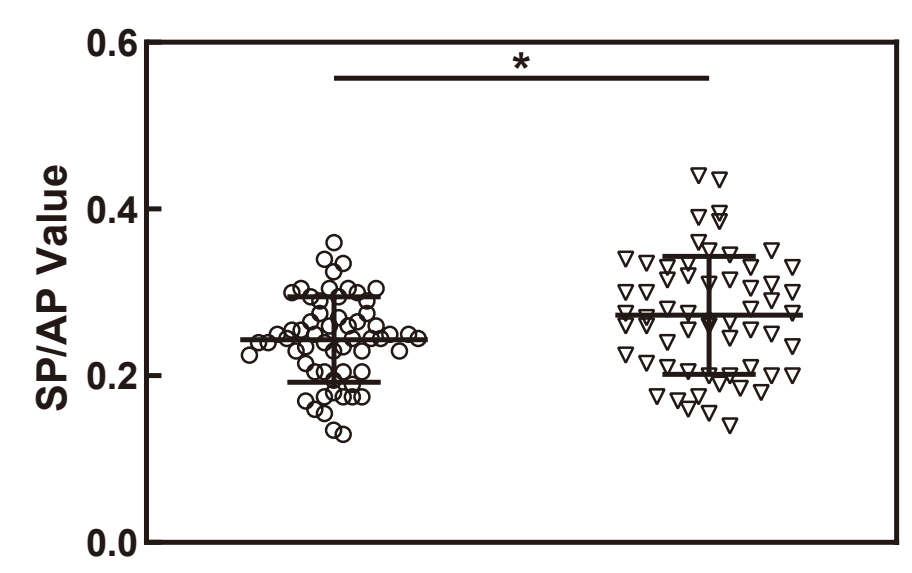

\section{Worse SPIAP}

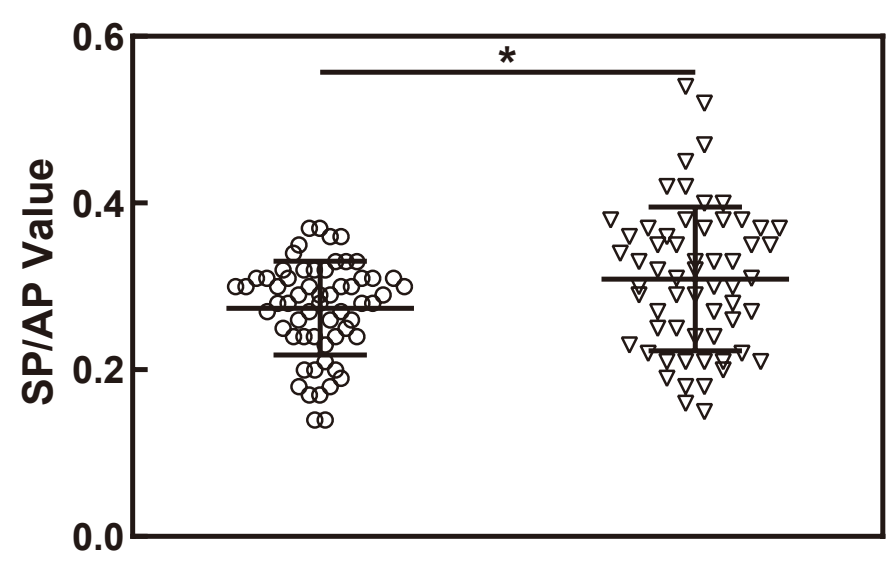

Handedness Side SPIAP

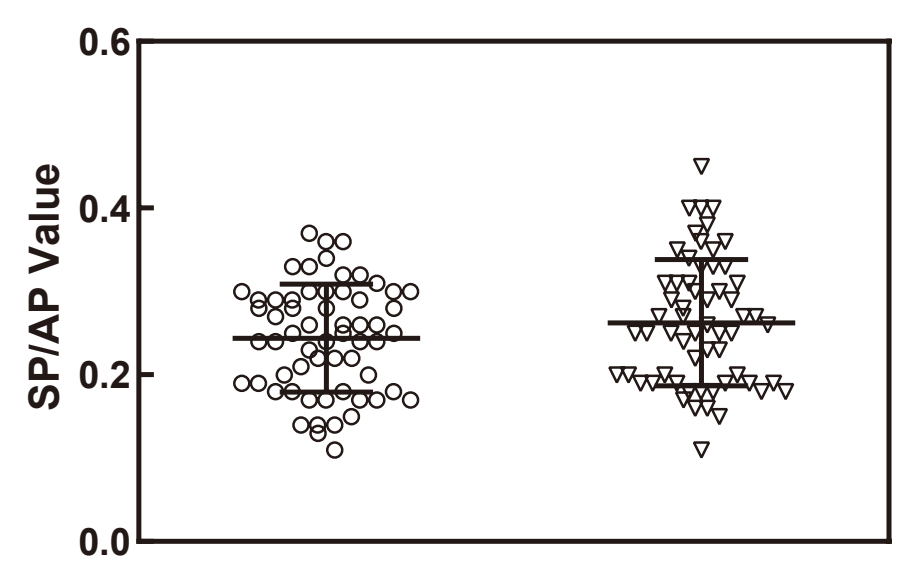

- Control

$\nabla$ HHL Risk 
medRxiv preprint doi: https://doi.org/10.1101/2020.09.27.20202481; this version posted March 25, 2021. The copyright holder for this preprint (which was not certified by peer review) is the author/funder, who has granted medRxiv a license to display the preprint in perpetuity.

All rights reserved. No reuse allowed without permission.

Table 1. Correlation analysis between the speech-in-noise scores and electrocochleography values

SP/AP Value

\begin{tabular}{lccc}
\cline { 3 - 3 } & & & Handedness \\
& & Woan & Side \\
\hline Pearson correlation & -0.207 & -0.198 & -0.131 \\
P value & $0.023^{*}$ & $0.030 *$ & 0.154 \\
\hline
\end{tabular}

The $\mathrm{p}$ values less than 0.05 are indicated*. 
medRxiv preprint doi: https://doi.org/10.1101/2020.09.27.20202481; this version posted March 25, 2021. The copyright holder for this preprint (which was not certified by peer review) is the author/funder, who has granted medRxiv a license to display the preprint in perpetuity.

All rights reserved. No reuse allowed without permission.

Table 2. Correlation analysis between the gap thresholds and electrocochleography values

SP/AP Value

$\begin{array}{llll}\text { Pearson correlation } & 0.132 & 0.092 & 0.125 \\ \text { P value } & 0.151 & 0.317 & 0.175\end{array}$

$2 \mathrm{kHz}$ Marker

$\begin{array}{llll}\text { Pearson correlation } & -0.112 & -0.106 & -0.115 \\ \text { P value } & 0.228 & 0.253 & 0.214\end{array}$

$1 \mathrm{kHz}$ Marker

$\begin{array}{llll}\text { Pearson correlation } & -0.067 & -0.034 & 0.005 \\ \text { P value } & 0.471 & 0.714 & 0.954\end{array}$

